\title{
Modèle réduit physique de la Rivière des Galets (La Réunion)
}

\author{
J. Duvoisin
}

SOGREAH

\begin{abstract}
Ce modèle a été construit afin de définir, avec de meilleures garanties, diverses solutions d'endiguement du cône de la rivière compte tenu des conditions sévères naturelles: pente $2 \%$, débit centennal $2400 \mathrm{~m}^{3} / \mathrm{s}$, transport par charriage important de matériaux présentant un large étalement granulométrique (de $50 \mu$ à $1 \mathrm{~m}$ ), prise en compte de l'effet des extractions de matériaux, etc...

L'importance et la qualité des moyens mis en œuvre sur le modèle ont permis des observations et des conservations de données expérimentales sur disquettes qui constituent une base de données d'un grand intérêt dans le domaine du comportement des lits des rivières torrentielles alluviales à fort charriage.
\end{abstract}

\section{Les données nature}

Il est utile de situer le niveau de connaissance des données naturelles et des études préalables :

Granulométrie : Expoitation de 140 échantillons prélevés dans les alluvions récentes de la rivière suivant 12 profils en travers répartis sur $6 \mathrm{~km}$ de longueur de rivière (année 1977). Après réalisation et contrôle de 20 nouveaux prélèvements en 1983, détermination du fuseau granulométrique représentatif du matériau naturel et du fuseau du matériau modèle (fig. 1).
Hydrologie : A partir des relevés pluviométriques disponibles et des indications sur les niveaux atteints par les crues cycloniques "Denise " en 1966 et " Hyacinthe " en 1980, reconstitution de la chronique de l'ensemble des épisodes de crues de la rivière de 1951 à fin 1982. C'est cette chronique de débits réels qui permettra la constitution des cycles d'histogrammes des crues pour l'étalonnage et le réglage du modèle.

Topographie: En 1983, réalisation d'un levé topographique à l'échelle $1 / 2000$ de l'ensemble du lit représenté sur le modèle entre la cote 150 et la mer, soit $7,5 \mathrm{~km}$ de longueur de rivière pour un lit actif couvrant une superficie totale de l'ordre de 500 hectares. Restitution de la topo-

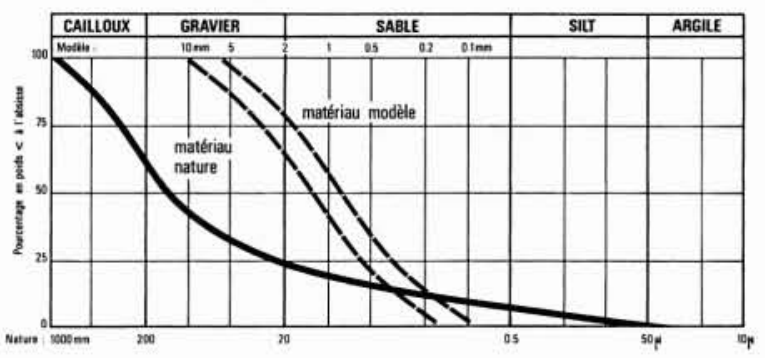

1 Faisceaux granulométriques modèle et nature.

\section{Scale model of the " rivière des Galets" (Réunion Island)}

This model was designed in order to define with better guaranties various solutions of banking in of the river cone, considering hard natural conditions : $2 \%$ slope, $2400 \mathrm{~m}^{3} / \mathrm{s}$ centennial flow, large bed load, transport of materials with a wide granulometric spreading out (from $50 \mu$ to $1 \mathrm{~m}$ ), taking into account the effect of materials extraction, and so on. Observations and storage on diskettes of experimental data which are of great interest with regard to the behaviour of alluvial torrential rivers with a large bed load, are the result of important and hight-quality means implemented on the model. 




2. Schéma synoptique du système.

graphie de 1966 après le passage du cyclone Denise, à partir d'une couverture aérienne IGN, par dix profils en travers caractéristiques de la zone d'étude. Pour des raisons de précision de restitution, il n'a pas été possible d'utiliser la couverture aérienne de 1951.

\section{Le modèle réduit}

Construit dans un hangar de $3000 \mathrm{~m}^{2}$ consacré à cette étude et implanté sur la zone industrielle $\mathrm{n}^{\circ} 2$ de la commune du Port à La Réunion, ce modèle représentait les 7,5 derniers kilomètres de la Rivière des Galets.

La partie amont du lit était limitée latéralement par des berges en dur ou des terrasses anciennes consolidées, mais sur les trois derniers kilomètres, le vaste cône, comme dans la nature, n'était pas limité. Les limites du modèle laissaient les chenaux divergents se développer sans contraintes jusqu'à $1600 \mathrm{~m}$ de largeur à l'estran.

\section{Les mesures et le traitement des données (fig. 2)}

Outre les dimensions exceptionnelles du modèle $(70 \mathrm{~m}$ de long sur une largeur de 10 à $15 \mathrm{~m}$ ), l'intérêt et l'originalité de cette installation ont résidé, d'une part, dans l'utilisation de nouveaux types d'appareils de mesure et, d'autre part, dans la constitution d'un fichier systématique de l'ensemble des opérations et des mesures effectuées sur le modèle.

L'ensemble du système de mesure était organisé autour d'une centrale de mesure HP 3497 A commandée par un ordinateur IBM PC-XT.

Concernant les aspects primordiaux de cette étude, le transport solide et l'évolution morphologique du lit, deux appareils doivent être mentionnés : la balance doseuse différentielle DIW 107-400, qui assurait l'alimentation en débits solides avec une précision de $\pm 5 \%$, le capteur de niveaux d'eau et de fond (Limnidyn) créé pour la circonstance.

Une série initiale, complétée en cours d'essais, de 14 programmes informatiques totalisant 20000 instructions, assurait le contrôle des processus hydrauliques et morphologiques du modèle, la commande entièrement automatique des capteurs Limnidyn pour assurer les relevés des fonds sur la totalité des profils, la présentation des résultats des mesures au moyen de tracés et de tableaux imprimés.

Il faut signaler en outre, et contrairement aux installations habituelles de ce type d'études, que le matériau solide ayant transité sur le cône n'était pas recyclé (il s'agit d'un matériau alluvionnaire de densité 2,7 , broyé et recomposé pour respecter le faisceau granulométrique prévu). A intervalles constants et comparatifs d'essais, le matériau qui se décantait à l'aval était séché, pesé et évacué à l'extérieur du hangar.

\section{Les données recueillies et disponibles (fig. 3)}

Le tableau de la figure 3 donne une indication sur le type de données disponibles et leur densité. Ces données existent :

- pour 5 cycles répétitifs d'un histogramme de crues entre 1966 et 1983 (comparaison possible avec l'évolution de la topographie nature) où ont varié la relation débit liquide/débit solide et la constitution du mélange d'alimentation des matériaux solides (coupure des catégories les plus grosses pour les faibles débits). Pour les trois premiers cycles; les mesures étaient limitées à la zone amont du modèle muni, pour l'occasion, de deux profils supplémentaires de mesures;

- pour trois cycles répétitifs d'un histogramme "prospectif » de crues sur 60 ans environ de vie de la rivière, avec des mesures sur la totalité du modèle.

Pour la partie amont du modèle qui, pour l'ensemble des opérations, est restée "non aménagée ", il est possible de disposer des données portant sur 12 cycles de l'histogramme "prospectif ».

\section{Commentaires}

\subsection{Les moyens mis en auvre et les résultats acquis}

L'élaboration et la mise en œuvre de moyens d'investigation aussi importants étaient justifiées par la puissance exceptionnelle des phénomènes naturels (pente moyenne du lit de $2 \%$ dans la zone de l'étude, débit centennal de $2400 \mathrm{~m}^{3} / \mathrm{s}$ pour un bassin versant de $100 \mathrm{~km}^{2}$, charriage de l'ordre de $1 \%$ du débit liquide en poids pour les débits supérieurs à la crue annuelle) et par l'importance de l'enjeu. Rappelons par ailleurs que le modèle a été construit et exploité à $10000 \mathrm{~km}$ de la Métropole. 
L'exploitation des résultats acquis sur le modèle a permis de concevoir et de dimensionner les caractéristiques hydrauliques des ouvrages de défense avec un maximum de fiabilité et de sécurité. C'était l'objectif principal de cette étude, compte tenu des conséquences catastrophiques, pour les ouvrages et les riverains, qui résultent en général du passage des crues cycloniques.

\subsection{Les compléments d'équipements et les enseignements plus généraux}

En cours d'étude, il est apparu que la somme importante de résultats pouvait être exploitée dans un esprit plus large de recherche pour une meilleure connaissance des lois de la morphologie fluviale de ce type de rivière. Il n'a pas été possible cependant de trouver à ce moment les moyens nécessaires pour mettre en œuvre les aménagements de l'équipement du modèle afin de compléter et de prolonger les résultats acquis. L'éloignement de la métropole et le défaut de spécialisation locale de l'Université n'ont pas permis aux démarches entreprises de déboucher en temps utile. En outre, l'équipe chargée de l'exploitation du modèle n'avait pas les moyens adaptés à ce type de recherche appliquée.

Ce modèle est maintenant détruit. Il reste cependant en mémoire de l'ordinateur des informations nombreuses et originales qui mériteraient d'être exploitées. Dans les domaines concernant le transport solide, le pavage et le tri granulométrique notamment, cette modélisation d'un large étalement granulométrique des matériaux avec un circuit d'alimentation "ouvert " sans recyclage devrait apporter

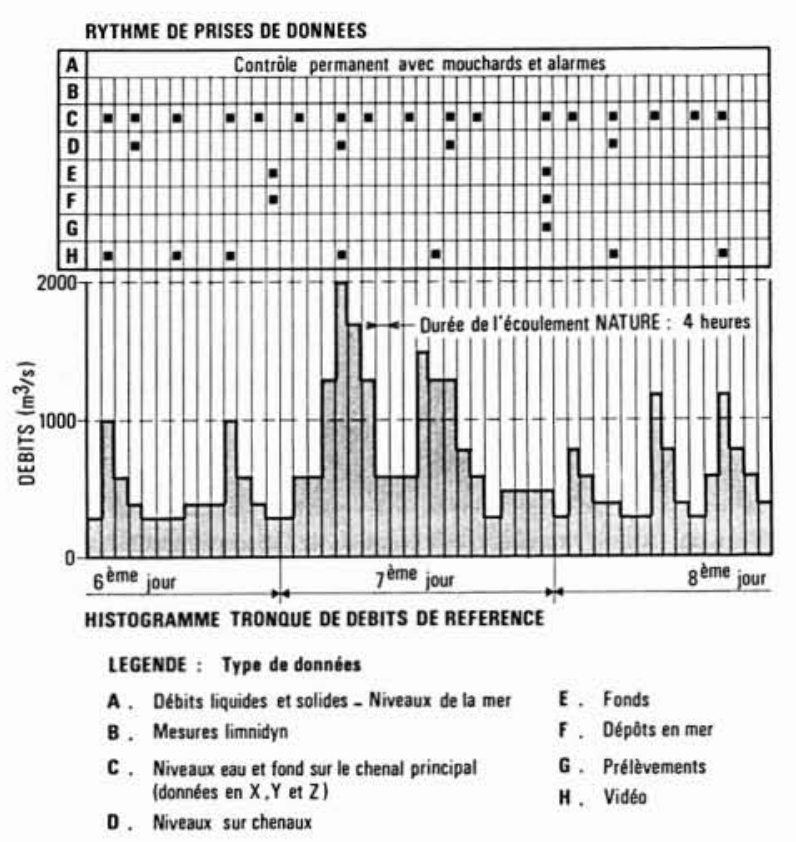

3. Une séquence d'essai. des éléments à la réflexion pour la recherche d'une meilleure connaissance de ces phénomènes complexes.

Bien qu'elles n'aient pas été conçues et traitées à cet effet, les mesures effectuées devraient apporter des éléments exploitables pour les travaux entrepris notamment sur la mise au point de modèles numériques de représentation d'évolution des lits de rivière (1) et l'approche de formulation de loi de morphologie fluviale adaptées au cas (2).

\subsection{Observations}

Les rapports des essais et les rapports successifs des " experts" qui suivaient le déroulement de l'étude constituent, notamment pour les phases de réglage et d'étalonnage du modèle, un ensemble de réflexions et d'observations concernant le transport solide, le tri granulométrique, le pavage, ...

\section{Transport solide - Charriage}

Une loi moyenne débit liquide/débit solide en fin des phases de réglage a été adoptée pour l'alimentation du modèle, mais il reste que la meilleure garantie d'illustrer correctement le phénomène réside dans le fait d’avoir représenté une grande longueur de lit à l'amont de la zone que l'on devait aménager.

Sur un tronçon de rivière, la relation débit liquide/débit solide nous paraît tributaire, à un instant donné, des épisodes de crues qui ont précédé.

Dans la zone médiane du modèle, nous avons observé des fluctuations passagères des fonds moyens du lit qui correspondaient à des déplacements de volumes de matériau sans comparaison avec les volumes injectées à l'amont du modèle.

Ces phases d'embâcle de matériaux (qui ne sont pas, dans notre cas, du type lave torrentielle) ou, au contraire, de dégraissement localisé du lit, sont couramment constatés sur les rivières de La Réunion.

Il existe pour les services responsables des «crues d'eau claire " et des "crues de matériau "; les deux dépressions successives de février 1987 sur les rivières Nord-Est de l'île ont été décrites comme telles dans les rapports officiels.

\section{Pavage et tri granulométrique}

Dans la nature, et sur la totalité du tronçon représenté sur le modèle, la granulométrie du matériau est très homogène; nous constatons la même proportion de gros blocs à l'approche de la mer que $7 \mathrm{~km}$ à l'amont.

Le matériau injecté à l'amont au cours des premières phases de réglage correspondait, pour tous les débits, au fuseau granulométrique du matériau constitutif du lit de la rivière (3).

Lors des séquences de faibles débits, entre 300 et $700 \mathrm{~m}^{3} / \mathrm{s}$, on a constaté un phénomène sensible de grossissement du fuseau granulométrique des matériaux dans le lit, bien que les constatations premières situaient le débit de charriage généralisé autour de $350 \mathrm{~m}^{3} / \mathrm{s}$ dans un chenal unique d'écoulement. 
Ce phénomène intéressait la zone amont du modèle, où l'on notait des atterrissements détectés à la fois par les relevés de profils en travers et par les pentes d'écoulement dans les chenaux.

Pour la suite des essais, on a modifié la granulométrie du matériau injecté dans le modèle. Une coupure de l'ordre de $70 \%$ de la catégorie $4 / 8 \mathrm{~mm}$ (soit 0,5 à $1 \mathrm{~m}$ nature) par tamisage sur grille à la sortie de la balance doseuse a permis de supprimer cette tendance.

Lors des derniers essais de réglage et d'étalonnage du lit, les prélèvements effectués ont confirmé les constatations d'homogénéité de granulométrie sur l'ensemble du tronçon de rivière.

Les matériaux qui transitent sur le modèle comportent une plus forte proportion d'éléments fins comparés au matériau constitutif du lit (comparaison des apports recueillis à l'aval du modèle avec le fuseau de référence). Cette tendance est très nette pour les séries de faibles débits; elle est fortement atténuée lorsque l'histogramme des débits comporte, conformément à la nature, des séquences de forts débits.

Une tendance au pavage des fonds des chenaux a été mise en évidence (4) lorsque, pour des raisons particulières, ces chenaux restaient fixés malgré les changements du régime des crues.

C'est le cas à l'état naturel en tête d'un éperon rocheux ou dans un rétrécissement ; c'est le cas pour des endiguements qui limitent le balayage latéral du cône alluvial.
Ce phénomène n'était pas détectable lorsque les chenaux divaguaient librement pour balayer l'ensemble du cône.

On a pu observer, dans ce cas, que le fond des chenaux avait tendance à se paver lors de séries de faibles débits liquides, pratiquement sans charriage apparent sur le modèle et dont les écoulements occupaient des lits façonnés par de fortes crues antérieures.

Lors de nouveaux changements de régime, l'ensemble du chenal était de nouveau occupé, et nous observions un charriage généralisé sur l'ensemble du fond sans pouvoir distinguer ce qu'il advenait de l'amořce de pavage. Par la suite, les écoulements vifs abandonnaient le plus souvent ces chenaux au bénéfice de nouveaux cheminements ; les fonds étaient alors recouverts par des atterrissements soit dans le même temps, soit à l'occasion de nouveaux changements du régime des écoulements.

(1) Travaux de MM. Bouvard et Chollet (Institut de Mécanique de Grenoble). Thèse de J.-P. RAHUEl (SOGREAH-IMG).

(2) Travaux de M. Rametre: M. Ramette a suivi comme expert du Maître d'Euvre l'ensemble des phases d'exploitation du modèle de la Rivière des Galets.

(3) Le fuseau adopté correspondait au fuseau représentatif des sections dynamiques de la zone de l'étude.

(4) Par examen des courbes granulométriques des matériaux prélevés sur l'ensemble de la section vive ayant participé aux écoulements.

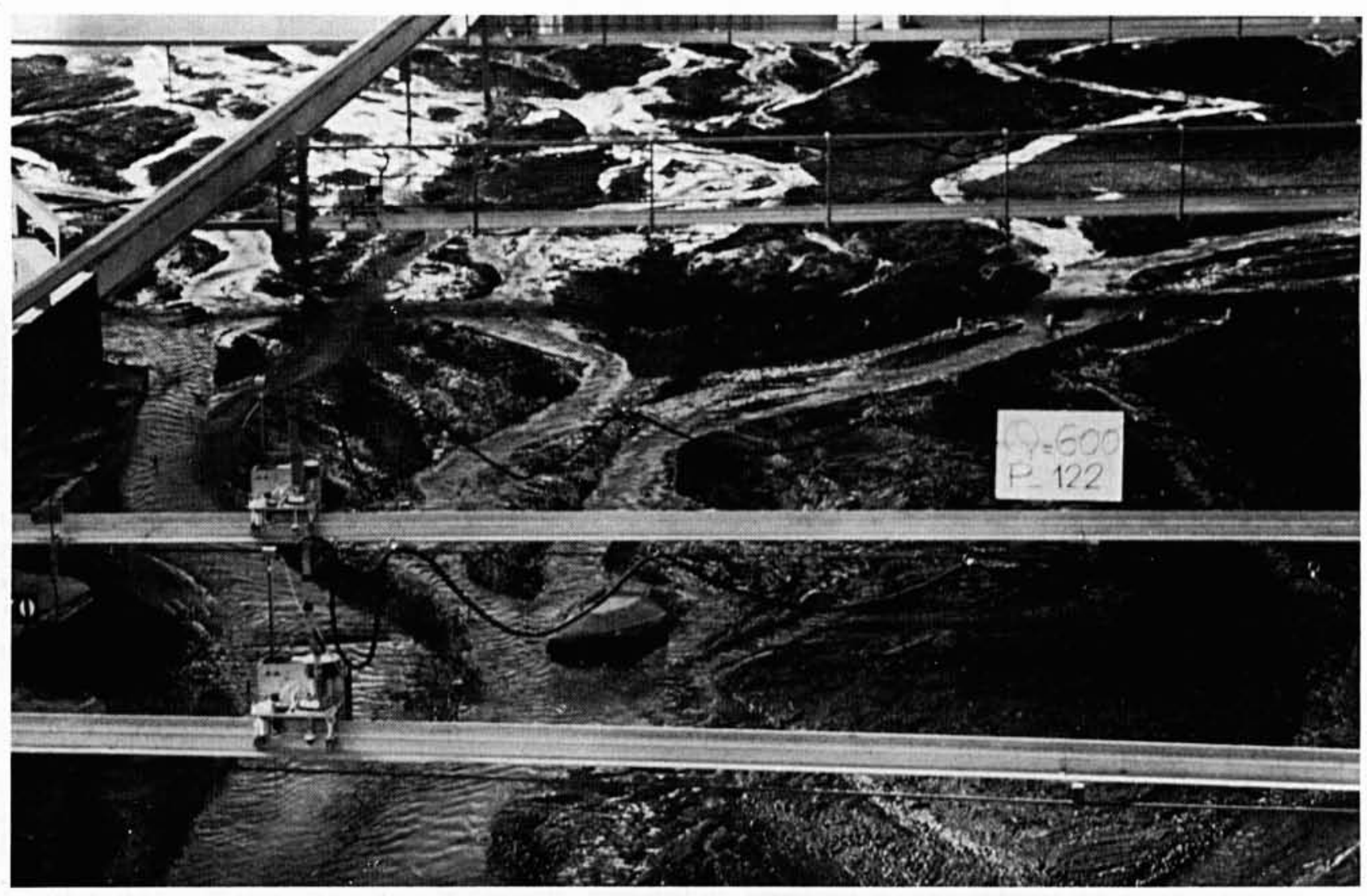

